\title{
NON-EQUILIBRIUM CONDITION IN THE SNR
}

\author{
H. TSUNEMI \\ Graduate School of Science, Osaka University \\ 1-1 Machikaneyama-cho, Toyonaka, Osaka 560, Japan
}

\section{Introduction}

SNe are one of the major source of the heavy elements in Galaxy. They produced high temperature plasma heavily contaminated with heavy elements mixing with the interstellar matter. Due to the temperature and the density, the shock heated plasma does not reach the collisional ionization condition in young SNR, like Cassiopeia A, Tycho and Kepler. The emission is well represented with a non-equilibrium condition plasma.

ASCA observed these young SNR with its high energy resolving power. We could detect three types of emission lines from $\mathrm{Si}, \mathrm{S}: K \alpha$ from He-like ion, $K \beta$ from $\mathrm{He}$-like ion and $K \alpha$ from $\mathrm{H}$-like ion. The intensity ratios between these lines, we could determine the non ionization degree which is characterized by the ionization parameter, $\tau$, the product of the electron density and the time elapsed after the shock heating. With taking into account the density and the age of the young SNR, it makes sense that they do not reach the collisional equilibrium condition.

ASCA observed middle-aged SNR. The plasma in them are thought to reach the CIE condition with taking into account their ages. Furthermore, they gathered relatively large amount of interstellar matter which diluted the ejecta. The metal abundance there should have represented the average abundance of the interstellar matter. We report there the observational results of them.

\section{The Cygnus Loop}

The Cygnus Loop is a typical middle aged ( 18000years) SNR well studied in various wavelength. The $\mathrm{X}$-ray spectrum in the north-east rim clearly shows that the plasma does not reach the CIE condition (Miyata et al., 1994). The X-ray emitting plasma in the rim is always gathering the in- 


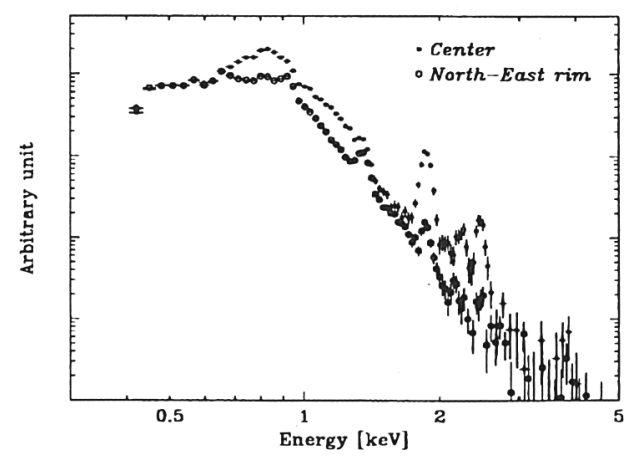

Figure 1. The SIS spectra for the north-east rim and for the center region of the Cygnus Loop. They are plotted by equalizing $O$ lines.

terstellar material. The newly coming interstellar materials are recently shock heated. The elapsed time after the shock heating has almost nothing to do with the SNR age. Therefore, it makes sense that they are fresh plasma showing NEI condition (Miyata 1995). The metal abundances in the north east rim are smaller than those of cosmic values. This fact, it is still puzzling, clearly supports that the X-ray emitting plasma in the rim is dominated not by the ejecta but by the interstellar matter.

The X-ray spectrum from the center portion of the Cygnus Loop consists of two components (Miyata et al., 1997). One is relatively low temperature showing clear $\mathrm{O}-\mathrm{K}$ lines. Line intensity ratio between $\mathrm{O}-\mathrm{VII}$ and $\mathrm{O}-\mathrm{VIII}$ shows almost the same value to that observed in the north-east rim. The other is relatively high temperature showing clear $\mathrm{Si}$ and $\mathrm{S}$ lines. The low temperature component must come from the rim region while $\mathrm{Si}$ and $\mathrm{S}$ lines come from the core region of the Loop which can be considered to be superposed to each other due to the projection effect. The plasma conditions in the core are metal rich, high temperature and low ionization parameter. The abundance of $\mathrm{Si}$ and $\mathrm{S}$ in the core region are 4 and 6 times those of cosmic values which are about 20-40 times higher than those observed in the north east rim. Figure 1 shows the X-ray spectrum of the core component. The apparent line widths of $\mathrm{Si}$ and $\mathrm{S}$ show 4 and 6 times those of cosmic values with a electron temperature of a few $\mathrm{keV}$.

The metal rich plasma in the core region of the Loop is relatively concentrated in a compact region. What is the origin of this metal rich plasma ? The straightforward interpretation is to understand it as fossil of the ejecta from the SN. The plasma component in the core region depends on 
the progenitor type. Based on the theoretical model (Thielemann et al., 1996), we can estimate the metal abundance. By comparing the mass of Si, $\mathrm{S}$ and $\mathrm{Fe}$, Miyata et al. (1997) claimed that the Cygnus Loop is originated from the type II SN. This is the first time that the SN type of the Cygnus Loop is determined from the metal abundance.

\section{The Vela SNR}

The Vela SNR is also a typical middle aged ( 10000years) SNR. There is an X-ray pulsar in its center confirming the type II origin. Far from the so called plerion type SNR, it has a relatively large shell showing a thermal emission.

ROSAT observation revealed that the Vela SNR is rather circular structure. Furthermore, it discovered that there are several cusp-shape structures outside the X-ray emitting shell. Aschenbach et al. (1994) claimed that they are the fragments produced in the SN explosion.

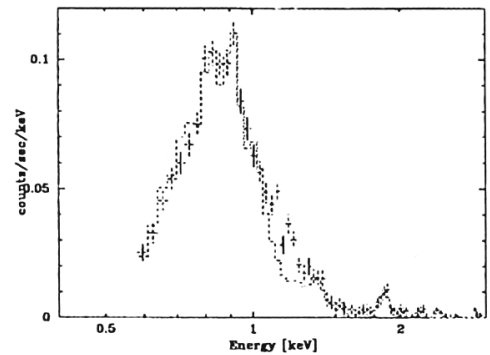

Figure 2. X-ray spectrum of the fragment $\mathrm{A}$.

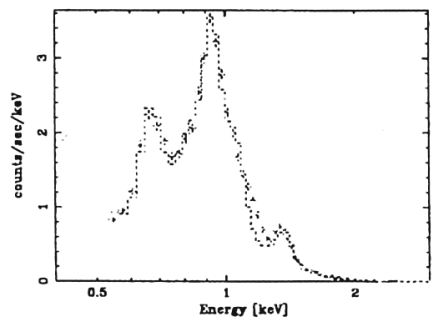

Figure 4. X-ray spectrum of the fragment $D$.

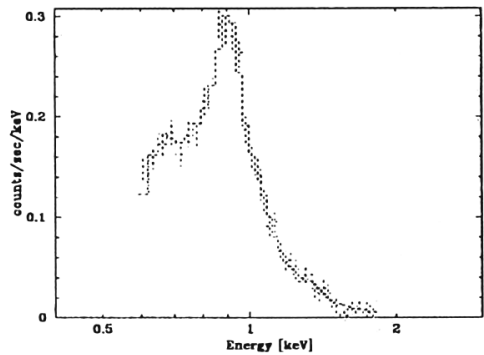

Figure 3. X-ray spectrum of the fragment B.

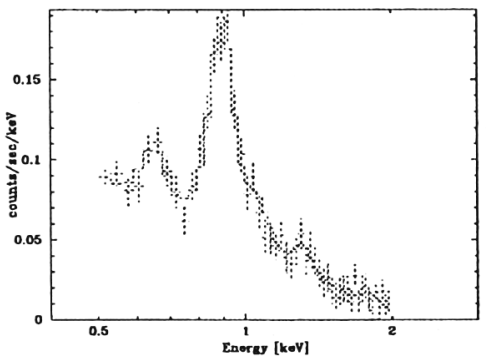

Figure 5. X-ray spectrum of the fragment $\mathrm{E}$.

ASCA observed so far four fragments out of six reported (A through F). They are the fragment, A, B, D and E. The other two are too dim to be detected by Asca. The X-ray spectra for them are shown in figures 2-5. Some are bigger than the FOV of the SIS which makes difficult to estimate the background level partly due to the radiation damage effect on the CCD. 
TABLE 1. The best-fit parameters by the NEI model.

\begin{tabular}{ccccccc}
\hline Fragment & $\mathrm{kT}(\mathrm{keV})$ & $O^{*}$ & $N e^{*}$ & $M g^{*}$ & $S i^{*}$ & $F e^{*}$ \\
\hline A & $0.31 \pm 0.02$ & 0.03 & 0.15 & 0.11 & 1.0 & 0.04 \\
B & $0.33 \pm 0.01$ & 0.09 & 0.49 & 0.14 & 0.03 & 0.05 \\
D & $0.36 \pm 0.02$ & 1.2 & 2.4 & 2.3 & 0.0 & 0.15 \\
E & $0.32 \pm 0.02$ & 0.08 & 0.33 & 0.15 & 0.11 & 0.17 \\
rim & $0.24 \pm 0.03$ & 0.02 & 0.07 & 0.11 & 0.08 & 0.01 \\
\hline
\end{tabular}

* Theses are the best fit values. The relative values are determined by $\pm 25 \%$ whereas the absolute values have large uncertainties.

Table 1 shows the result of metal abundance for them. Most of the spectra come from line emissions. Therefore, it is quite difficult to reach the absolute values for metal abundance. Whereas, the relative values among various elements are quite reliable. Keeping this point in mind, we notice that each fragment is characterized by one or two prominent elements. We also observed the shell region where we found relatively poor metal abundance. This reminds us the poor metal abundance in the shell region of the Cygnus Loop. Therefore, the metal abundance in the interstellar matter near the Vela SNR is much lower than those of the the fragments.

If they are the mixture of metal rich plasma with the interstellar matter, the metal rich plasma must be a debris of the progenitor star. It should have a so-called onion skin structure: each layer contains individual rich element. Therefore, the fragments may represent the constituent of some layer. It is quite interesting how they passed the shock front without being destroyed, since the shock front region is so dense.

The ASCA observation confirmed the NEI condition for the plasma condition in the young SNR. It also revealed that the plasma in the middle aged SNR does not reach the CIE condition. Furthermore, we detected possible fossils from them which might represent the metal abundance of the progenitor star of the SN.

\section{References}

Aschenbach, B., Egger, R. \& Trumper, J. 1995, Nature, 373, 587

Miyata, E., Tsunemi, H., Pisarski, R., \& Kissel, S. E. 1994, PASJ, 46, L101

Miyata, E., PhD thesis of Osaka university 1995

Miyata, E., Tsunemi, H., Kohmura, T., Suzuki, S., \& Kumagai, S. 1997, submitted

Thielemann, F.-K., Nomoto, K., \& Hashimoto, M. 1996, ApJ, 460, 408 